Method: A total of 110 patients after stroke (69 men and 41 women) with a mean age of $69.3 \pm 13.7$ years were recruited and completed (a) a sociodemographic-clinical questionnaire, (b) the Center for Epidemiological Studies-depression (CES-D) scale, and (c) Barthel Index.

Results: $76.4 \%$ of the participants had ischemic stroke; while the $76.4 \%$ were married and the $60 \%$ were retired. The $74.5 \%$ of our study participants were at risk for depression. $33.6 \%$ of our sample were receiving antidepressant treatment. Patients living alone had a higher depressive symptomatology score. Depression was significantly correlated negatively with patients' degree of functionality and independence. Of the participants in our sample, a percentage of $14.5 \%$ had received a disability pension, while $6.4 \%$ stated that they had no employment for health reasons, which is indicative of the disruption of their functioning.

Conclusions: The onset of depressive symptoms is common among survivors after stroke. Lonely living, as well as loss of functionality may lead to the appearance of depressive symptoms. Early detection and management of depression may facilitate patient's compliance to the rehabilitation program in order to achieve optimal therapeutic results and ensure a better quality of life.

\title{
525 - Anger expression among survivors after stroke in Greek elderly
} Afroditi Zartaloudi ${ }^{1}$, Anastasia Papadopoulou ${ }^{2}$, Panagiotis Papadopoulos ${ }^{2}$, Eirini Grammatopoulou ${ }^{1}$, Anna Kavga ${ }^{1}$

${ }^{1}$ University of West Attica, Athens, Greece

${ }^{2}$ Graduate of the post-graduate program "Neurological Diseases: Evidence based practice", University of West Attica, Athens, Greece

Introduction: Individuals may consider stroke complications as a threat to their well-being and selfesteem. Anger often occurs in patients after stroke and can disrupt the course of patient's recovery.

The aim of the present study was to investigate anger expression and degree of functionality in patients after stroke. Additionally, the relationship between the above parameters, as well as their correlation with socio-demographic characteristics and clinical factors of these patients were explored.

Method: A total of 110 patients after stroke (69 men and 41 women) with a mean age of $69.3 \pm 13.7$ years were recruited and completed (a) a sociodemographic-clinical questionnaire, (b) the State - Trait Anger Expression Inventory and (c) Barthel Index.

Results: $76.4 \%$ of the participants had ischemic stroke; while the $60 \%$ were retired. and the $20 \%$ of the sample showed severe to total dependence on self-care of basic parameters such as mobility, personal hygiene, feeding, incontinence and standing alone using the toilet. There was a positive statistically significant correlation between the degree of functionality and anger control. Patient, who were more independent and functional, exhibited more control of their anger. Women had statistically significant lower functionality/ independence score (BI) and anger-in score than men. Finally, patients with a history of prior stroke had higher anger-out score compared to patients with no previous history.

Conclusions: People with a previous history were more likely to suffer from greater and / or permanent functional impairment, disability, dependence, and experience emotional distress for longer periods of time. Women usually take care of other family members. Their inability to fulfill their role as well as their own dependence make the situation even more stressful, resulting in expressing anger. The results can be exploited by health professionals in order to recognize patients' difficulties in rehabilitation programs. 